\title{
Correction to: VPC: Pruning connected components using vector-based path compression for Graph500
}

\author{
Hao Bai ${ }^{1}$ (1) Xinbiao Gan ${ }^{1} \cdot$ Tianjing Xu ${ }^{1} \cdot$ Menghan Jia $^{1} \cdot$ Wen $\operatorname{Tan}^{1} \cdot$ Juan Chen ${ }^{1} \cdot$ Yiming Zhang $^{1}$
}

Published online: 15 November 2021

(c) China Computer Federation (CCF) 2021

\section{Correction to:}

CCF Transactions on High Performance Computing https://doi.org/10.1007/s42514-021-00070-z

In this article the following corrections have been made

- Removed the last keyword.

- In the 10th-13th line of the fifth paragraph of Section 1 Introduction, the last sentence should be "The mismatch between hardware and software designs becomes even more challenging (Wang et al. 2020)."

- In the 2nd-3rd line of the last paragraph of Section 1 Introduction, delete the sentence.

- Delete Section 3.

- In the Section 5 Parallel VPC customized with Tianhe, the 1st paragraph should be "Based on the serial VPC, we design parallel VPC in Tianhe, and we use multi-process and multi-thread to achieve parallelism."

The original article can be found online at https://doi.org/10.1007/ s42514-021-00070-z.

\section{Hao Bai}

baihao@nudt.edu.cn

Xinbiao Gan

xinbiaogan@163.com

Tianjing Xu

tianjingxu1021@163.com

Menghan Jia

jiamenghan12@nudt.edu.cn

Wen Tan

lingyingTW@163.com

Juan Chen

juanchen@nudt.edu.cn

Yiming Zhang

sdiris@gmail.com

1 College of Computer Science and Technology, National University of Defense Technology, Changsha 410073, China
- In Section 6.2 Experimental setup, the 1st paragraph should be "We have implemented VPC in Tianhe exascale prototype system."

- In 5th-6th line of the 3rd paragraph of Section 8 Conclusion and future work, it should be "Based on the serial VPC, we design parallel VPC in Tianhe."

The original article has been corrected. 